\title{
Emergency Monitoring System Based on a Newly-Developed Fall Detection Algorithm
}

\author{
Yun Jae Yi and Yun Seop Yü, Member, KIICE \\ Department of Electrical, Electronic and Control Engineering, Hankyong National University, Anseong 456-749, Korea
}

\begin{abstract}
An emergency monitoring system for the elderly, which uses acceleration data measured with an accelerometer, angular velocity data measured with a gyroscope, and heart rate measured with an electrocardiogram, is proposed. The proposed fall detection algorithm uses multiple parameter combinations in which all parameters, calculated using tri-axial accelerations and bi-axial angular velocities, are above a certain threshold within a time period. Further, we propose an emergency detection algorithm that monitors the movements of the fallen elderly person, after a fall is detected. The results show that the proposed algorithms can distinguish various types of falls from activities of daily living with $100 \%$ sensitivity and $98.75 \%$ specificity. In addition, when falls are detected, the emergency detection rate is $100 \%$. This suggests that the presented fall and emergency detection method provides an effective automatic fall detection and emergency alarm system. The proposed algorithms are simple enough to be implemented into an embedded system such as 8051-based microcontroller with 128 kbyte ROM.
\end{abstract}

Index Terms: Emergency monitoring, Fall detection, Sensitivity, Specificity

\section{INTRODUCTION}

The demand for ubiquitous healthcare is growing with rising medical costs incurred by the burgeoning elderly population [1].

The elderly, especially those above the age of 65 years, suffer falls owing to the deterioration of their physical functions. In Korea, $17.2 \%$ of the elderly above age 65 have suffered a fall at least once a year, and $23.7 \%$ of these have suffered a fall more than three times [2]. An emergency resulting from a fall-induced injury requires a rapid response. If the emergency response is not quick when an elderly person falls and becomes unconscious or is unable to move his/her body, he/she may succumb to any injuries that might have been caused by the fall [3]. Further, elderly persons who have experienced a fall could develop a fear of falling, leading to psychological anxiety and reduction in mobility, which causes balance, gait, and cognition disorders [4]. Thus, falls and the fear of falling are important issues that need to be tackled while monitoring the health of the elderly.

An emergency monitoring system is required to automatically detect a fall and to send an emergency alarm to the caregiver or the nearest healthcare center. The system for requesting emergency assistance can reduce the duration in which an elderly person remains unattended. Various types of emergency alarm systems have been reported [5-12]. The personal emergency response system (PERS) is a button that can be pushed to contact the emergency center by using wireless communication [5]. It has been found that the PERS was not used after most falls because of physical incapability or conscious unwillingness.

Received 28 February 2013, Revised 28 March 2013, Accepted 15 April 2013

*Corresponding Author Yun Seop Yu (E-mail: ysyu@hknu.ac.kr, Tel:+82-31-670-5293)

Department of Electrical, Electronic and Control Engineering, Hankyong National University, Anseong 456-749, Korea.

(c) This is an Open Access article distributed under the terms of the Creative Commons Attribution Non-Commercial License (http://creativecommons.org/li-censes/bync/3.0/) which permits unrestricted non-commercial use, distribution, and reproduction in any medium, provided the original work is properly cited. 
Various approaches to automatic fall detection by using accelerometers or gyroscopes have been proposed in recent years [6-15]. Automatic fall detection using a thresholdbased method of single parameters, which are calculated using acceleration measured by an accelerometer, has a high sensitivity (about 100\%), but relatively low specificity [610]. Automatic fall detection using multiple parameter combinations has a relatively high sensitivity (85.7\%) and specificity (90.1\%) [11]. Automatic fall detection using angular velocities measured by a gyroscope also have high sensitivity (100\%) and specificity (97.5\%) [12]. Further, automatic fall detection using multiple parameters, which are calculated using the acceleration and angular velocities measured by an accelerometer and a gyroscope, respectively, have high sensitivity (91\%) and specificity (92\%) [13]. However, because the reported fall detection algorithms do not have the desired sensitivity and specificity of $100 \%$, further development of advanced fall detection algorithms is required.

Automated fall detectors have been developed to ensure the independence and safety of the elderly. Most reported fall detection applications are prototypes or applications for research purposes [6-13] and commercial interests [14, 15]. However, even though falls are detected by the reported fall detection systems, the situation may not be an emergency and the fall may not cause any severe injury. Therefore, an algorithm is required to send an emergency alarm to the healthcare center in the case of an emergency when an elderly person falls.

In this paper, we propose an emergency monitoring system with a sensor node for detecting falls and resulting emergencies for the elderly, based on a newly developed fall detection algorithm. The sensor node consists of a ZigBee network processor chip (CC2530, Texas Instruments) and three types of sensors-an electrocardiogram (ECG), an accelerometer, and a gyroscope. The fall detection algorithm, which uses multiple parameter combinations calculated using the acceleration and angular velocity, is proposed. Further, we propose an emergency detection algorithm that monitors the movements and heart rate (HR) of the fallen elderly person, after the fall is detected. Various types of falls and activities of daily living (ADL) have been investigated with the proposed system for attaining the required sensitivity and specificity.

\section{MATERIALS AND METHODS}

A novel algorithm for monitoring an emergency situation based on falls using a sensor node is presented. The sensor node measures the acceleration, angular velocity, and HR with a tri-axial accelerometer, a bi-axial gyroscope, and ECG, respectively.

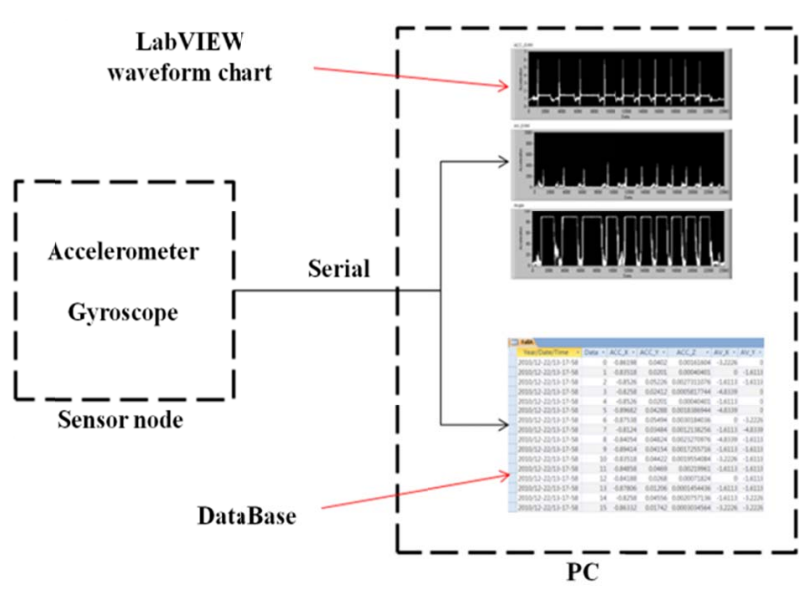

Fig. 1. Configuration diagram of the sensor node (that measures the acceleration, angular velocity, and heart rate) and the gateway (that monitors, calculates, and stores several parameters) in the fall detection algorithm.

Table 1. Test falls and activities of daily livings (ADLs) used in the study

\begin{tabular}{lrl}
\hline \multicolumn{2}{c}{ Content } & \multicolumn{1}{c}{ Activity } \\
& A & Running \\
ADL & B & Jumping \\
type & C & Sitting down and staniding up from a chair \\
& D & Lying down and standing up from a bed \\
& A & Falling forward over something \\
Fall & B & Falling laterally towards the side by losing balance \\
type & C & Sliding and falling backward \\
\hline
\end{tabular}

The sensor node is attached with an elastic belt at the chest. The sensor node, as shown in Fig. 1, measures sensors' data and sends them to the gateway (personal computer [PC]) by a ZigBee network processor. Emergency alarm simulation based on the fall detection algorithm is done in a virtual environment with a custom-made LabVIEW program [16].

\section{A. Subjects and Testing Activities}

Intentional falls were performed by three healthy volunteers: three male subjects whose ages ranged from 25 to 28 years; height, from 170 to $175 \mathrm{~cm}$; and weight, from 68 to $80 \mathrm{~kg}$. The falls were performed using a mattress (thickness, $20 \mathrm{~cm}$ ). Each subject performed three types of falls and four types of ADLs, as listed in Table 1. A total of 201 ADLs and 152 falls were tested. The ADLs used in this study were the activities that may have caused high impact or abrupt changes in a person's movement-and thus possibly resulted in a false alarm being triggered by the thresholdbased fall detection algorithm [7, 12]. 


\section{B. Hardware Description}

The ECG sensor is used for measuring the HR and consists of three commercial electrodes, an analog front endincluding an instrument amplifier (AD620; Analog Devices, Norwood, MA, USA) and an anti-aliasing filter, and a digital signal processing part-that uses a 16-bit microcontroller (dsPIC30F3012; Microchip Technology Inc., Chandler, AZ, USA). The analog front end amplifies the raw signals from the three electrodes, restores the variation caused in the DC components by using an analog integrator, and low-pass filters the raw signals with a cut-off frequency of $250 \mathrm{~Hz}$ by using an anti-alias filter. The variation in the DC components is caused by the variation in the contact resistances between the electrodes and skin. The dsPIC30F3012 (Microchip Technology Inc.) samples the output analog signals from the analog front end to 12-bit digital signals and samples the raw signals with $1 \mathrm{kHz}$ in this system. It also filters the digitally sampled signals using a designed infinite impulse-response band-pass filter in the frequency range 6 to $30 \mathrm{~Hz}$. To develop the algorithm for calculating the HR, the sensing data are transmitted to the gateway that monitors the ECG data and calculates the HR using LabVIEW, as shown in Fig. 2. The $\mathrm{HR}$ is calculated from the R-R interval measured from the ECG sensor [17].

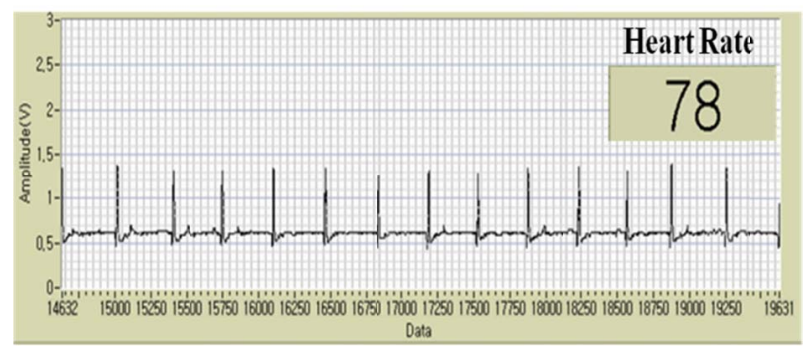

Fig. 2. Electrocardiogram data sent from the sensor node and heart rate calculated with LabVIEW.

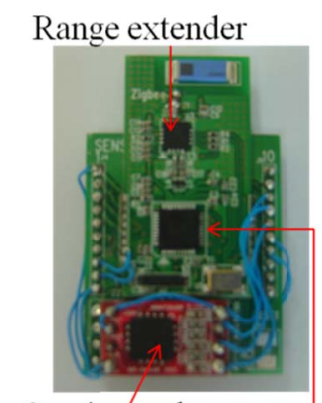

3-axis accelerometer $\mathrm{RF}$ transceive

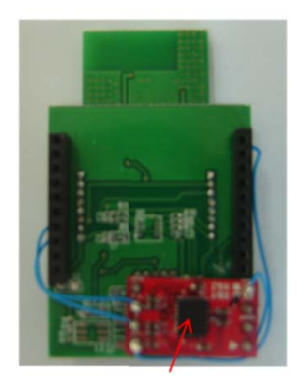

2-axis gyroscope (a)

(b)

Fig. 3. Photograph of the sensor node $(20 \mathrm{~mm} \times 50 \mathrm{~mm})$ for fall detection: (a) front and (b) back. RF: radio frequency.
Falls suffered by the elderly wary in terms of acceleration and angular velocity. The fall detection sensor consists of a \pm 6 g tri-axial accelerometer (MMA7260; Freescale Semiconductor, Austin, TX, US.A) and a $\pm 2,000 \%$ s bi-axial gyroscope (IDG-650; InvenSense, San Jose, CA, USA), as shown in Fig. 3. The sensor is controlled by the ZigBee network processor. The sampling rate is set to $100 \mathrm{~Hz}$, a bandwidth exceeding the characteristic response of human movement. Each tri-axial acceleration and bi-axial angular velocity was statistically calibrated in order to correct any possible axis tilt due to the orientation of the device on the subject or due to lower back tilt of the subject [18]. To develop the algorithm that detects falls suffered by an elderly person, the gateway monitors the accelerations and angular velocities sent from the sensor nodes and calculates several parameters such as the directions, magnitude, and angles of the elderly person's motion from the sensing data using LabVIEW in real-time, and stores the measured data and calculated parameters in an MS Access database, as shown in Fig. 1.

\section{Parameters}

To detect a fall and an emergency, five types of parameters are used in the analyses. Three different parameters- $A V_{S V M}$ (total sum vector of the angular velocities), $A C C_{S V M}$ (total sum vector of the accelerations), and $m$ Angle (mean of the tilted angles from the vertical of $n$ samples)-are used for detecting falls. Two different parameters- $m A C C_{p p}$ (mean peak-to-peak acceleration of $m$ samples below ith sample) and $m A V_{p p}$ (mean peak-to-peak angular velocity of $m$ samples below ith sample)—are used for detecting an emergency after a fall.

$A V_{S V M}$ and $A C C_{S V M}$ are calculated as follows [13]:

$$
\begin{gathered}
A V_{S V M}(i)=\sqrt{X_{a v}^{2}(i)+Y_{a v}^{2}(i)}, \\
A C C_{S V M}(i)=\sqrt{X_{a c c}^{2}(i)+Y_{a c c}^{2}(i)+Z_{a c c}^{2}(i)},
\end{gathered}
$$

where $i$ denotes the sample number; $X_{a v}(i)$ and $Y_{a v}(i)$, the angular velocity ( $\%$ ) of ith sample in the $x$ and $y$ directions, respectively; and $X_{a c c}(i), Y_{a c c}(i)$, and $Z_{a c c}(i)$, the acceleration $(g)$ of ith sample in $x, y$, and $z$ directions, respectively. The Euler angle $\theta$ denotes the tiltied angle between the accelerometer $z$-axis and the vertical, which is calculated from the accelerations in the three directions [19]:

$$
\theta(i)=\tan ^{-1}\left(\frac{\sqrt{Y_{a c c}^{2}(i)+Z_{a c c}^{2}(i)}}{X_{a c c}^{2}(i)}\right) \times \frac{180}{\pi} .
$$

$m$ Angle is calculated from the calculated tilted angles of $n$ samples as 


$$
m \text { Angle }(i)=\frac{1}{n}\left(\sum_{j=0}^{n-1} \theta(i-j)\right)
$$

where $n$ is the number of samples and $j$ is the sample number. $m A C C_{p p}$ and $m A V_{p p}$ are calculated as follows:

$$
\begin{array}{r}
m A C C_{p p}(i)=\frac{1}{m}\left(\sum_{j=0}^{m-1} A C C_{p p}(i-j)\right) \\
m A V_{p p}(i)=\frac{1}{m}\left(\sum_{j=0}^{m-1} A V_{p p}(i-j)\right)
\end{array}
$$

where $m$ is the number of samples for $A C C_{p p}$ and $A V_{p p}$.

\section{Emergency Alarm Algorithm}

When an elderly person falls, he/she may become unconscious or may be unable to move his/her body. Therefore, accurate emergency detection after detecting a fall is needed. The emergency alarm algorithm is shown in Fig. 4. First, the accelerations, angular velocities, and ECGs are measured and the parameters are calculated. Second, the fall detection algorithm, as shown in Table 2, is applied to detect a fall.

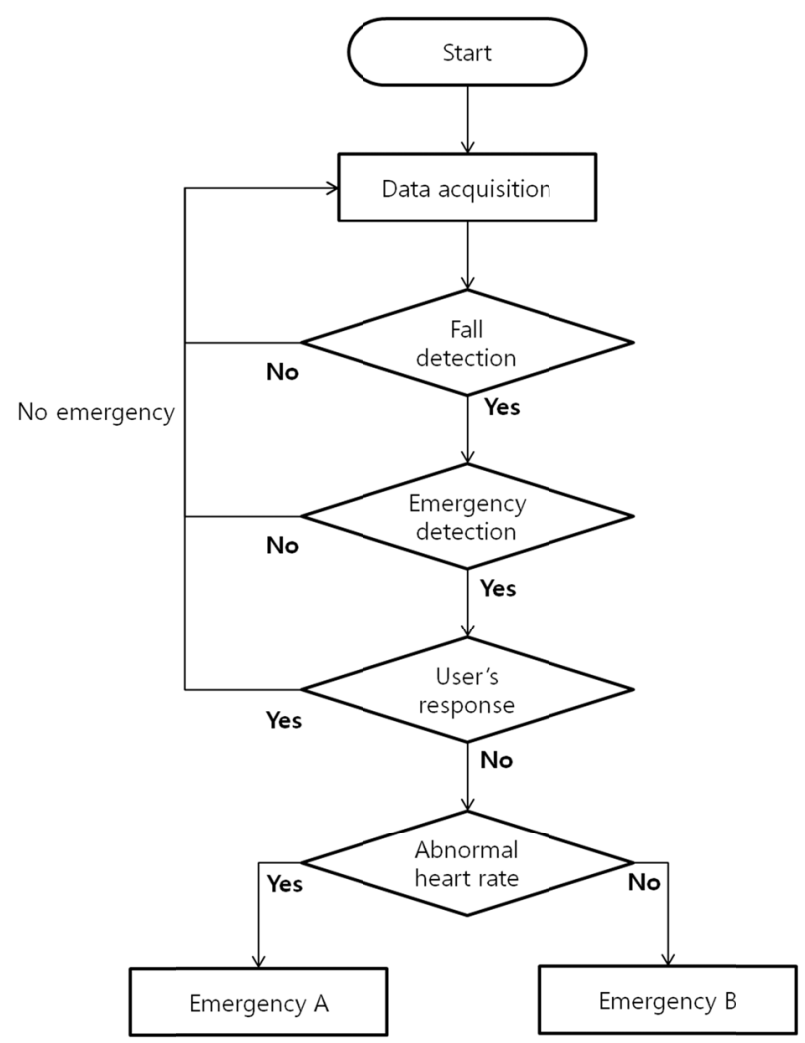

Fig. 4. Flowchart for the emergency alarm based on fall detection algorithm.
Table 2. Fall detection algorithm

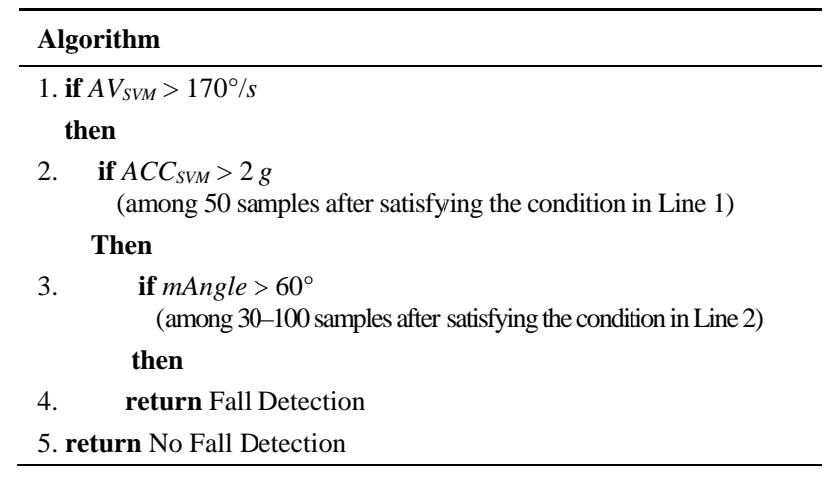

Table 3. Emergency detection algorithm

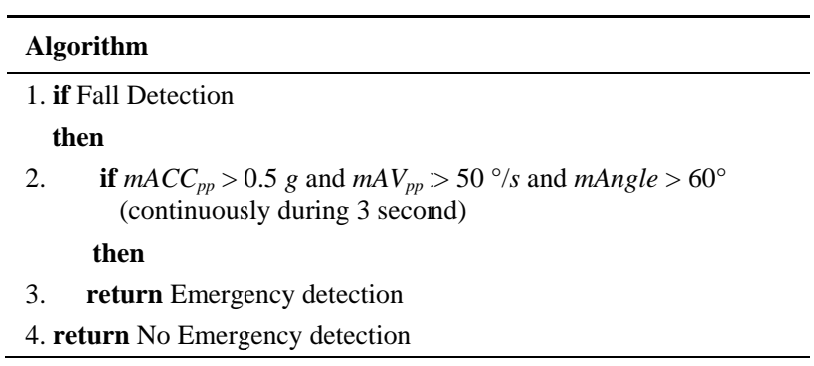

Third, if a fall is detected, the emergency detection algorithm, as shown in Table 3 , is applied to detect any emergency in which the faller may be unconscious or unable to move. Fourth, if an emergency is detected, the gateway addresses the elderly person by using a voice message to clarify whether he/she needs emergency assistance. If there is no response, two types of emergency alarms (A or B) are sent to the healthcare center or caregivers. According to the HR obtained, either emergency signal A, for an abnormal HR, or emergency signal B, for a normal HR, is sent.

\section{E. Fall Detection Algorithm}

For each parameter, the best threshold for discriminating between normal gait and falls is determined by plotting the range of possible thresholds of the parameters using the receiver operating characteristic curve [7, 12]. Figs. 5 and 6 show the $A C C_{S V M}$ curves measured for four types of ADLs and three types of falls, respectively. Tables 4 and 5 shows the maximum values of the parameters measured for four types of ADLs and three types; of falls, respectively. Here the numbers in parentheses refer to test numbers. Comparing the measured $A V_{S V M} \mathrm{~S}$ and $A C C_{S V M} \mathrm{~S}$ of the ADLs and falls, ADL-A and -B cannot be clearly distinguished from falls. In addition, comparing the measured mAngles of ADLs and falls, falls can be clearly distinguished from ADLs except for ADL-D. 


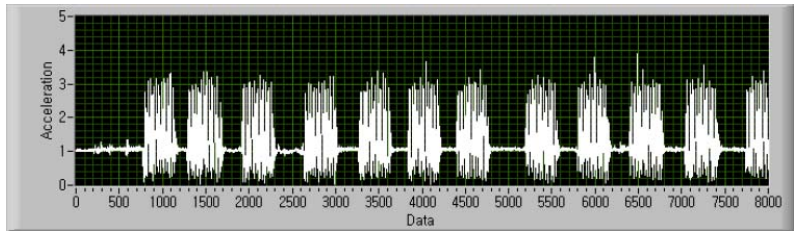

(a)

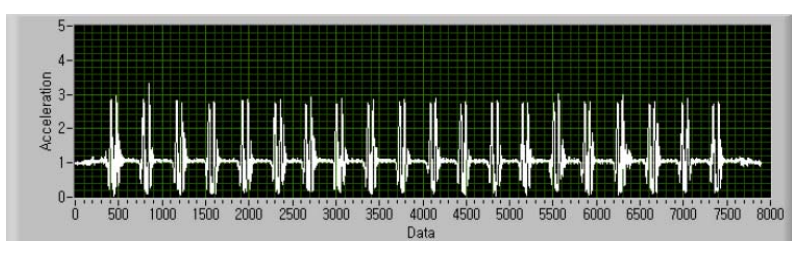

(b)

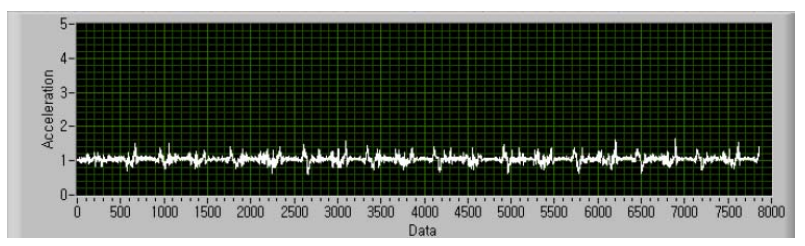

(c)

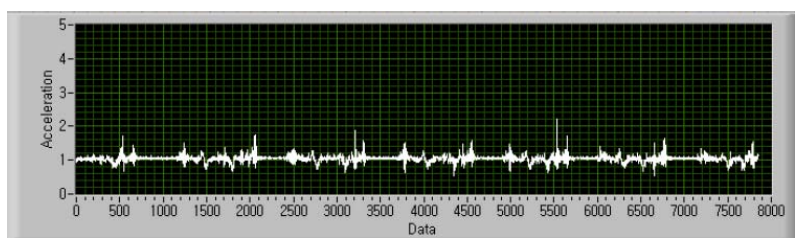

(d)

Fig. 5. $A C C_{S V M}$ measured for four types of activities of daily livings (ADLs): (a) ADL-A, (b) ADL-B, (c) ADL-C, and (d) ADL-D.

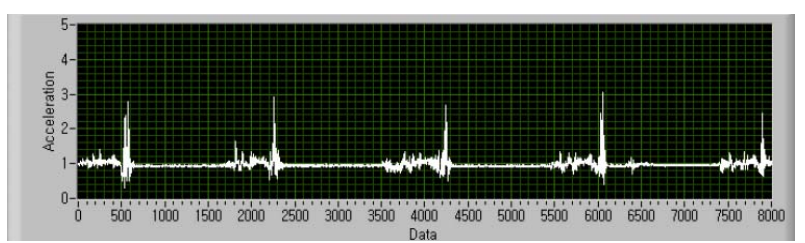

(a)

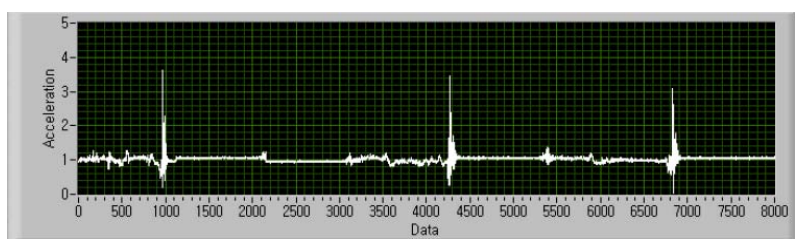

(b)

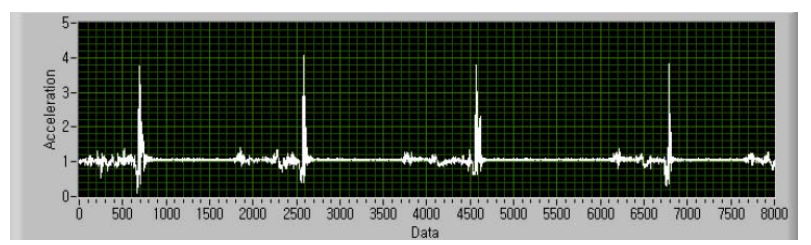

(c)

Fig. 6. $A C C_{S v m}$ measured for three types of falls: (a) fall-A, (b) fall-B, and (c) fall-C.
Therefore, ADL-A, -B, and -C can be discriminated from falls by using mAngle, and ADL-D can be discriminated from falls by using $A C C_{S V M}$. Firstly, the thresholds of $A V_{S V M}$ and $A C C_{S V M}$ are determined from the values discriminating between ADL-D and falls, and those of $m$ Angle are determined from the values discriminating between ADLs (A, B, and C) and falls. The thresholds determined from each measurement parameter are modified for the best specificity and sensitivity [7, 12]. The performance of the fall detection algorithm was studied separately for each parameter and for combinations of two or three parameters [11]. When studying multiple parameter combinations, the detection performance is tested when all the parameters are above certain thresholds within time intervals.

Because the acceleration and angular velocity during a fall do not vary synchronously, sometime interval (or scheduling) is needed to sense them. A fall detection algorithm using multiple parameter combinations with time scheduling is proposed, as shown in Table 2:

Line 1: Check whether the condition $A V_{S V M}>170 \%$ is satisfied.

Line 2: Check whether the condition $A C C_{S V M}>2 \mathrm{~g}$ is satisfied among the next 50 samples if the condition in Line 1 is satisfied.

Line 3: Check whether the condition mAngle $>60^{\circ}$ is satisfied among the next 30-50 samples if the condition in Line 2 is satisfied.

Line 4: Alarm fall detection, if the condition in Line 3 is satisfied.

Here, Lines 2 and 3 are the cases where time scheduling is applied.

Table 4. Maximum values of parameters measured at four types of activities of daily livings (ADLs)

\begin{tabular}{|c|c|c|c|}
\hline ADL type (no.) & $A C C_{S V M}(\mathrm{~g})$ & $A V_{S V M}(\% / s)$ & mAngle $\left({ }^{\circ}\right)$ \\
\hline$A(51)$ & 4.48 & 719.08 & 60.14 \\
\hline B (51) & 3.91 & 446.68 & 78.01 \\
\hline C (50) & 2.43 & 334.31 & 57.18 \\
\hline D (49) & 3.49 & 476.49 & 89.97 \\
\hline
\end{tabular}

Table 5. Maximum values of parameters measured at three types of falls

\begin{tabular}{cccc}
\hline Fall type (no.) & $\boldsymbol{A C C _ { S V M } ( \mathbf { g } )}$ & $\boldsymbol{A V _ { S V M } ( { } ^ { \circ } / \mathbf { s } )}$ & $\boldsymbol{m A n g l e}\left({ }^{\circ}\right)$ \\
\hline A (51) & 4.51 & 529.62 & 89.89 \\
B (50) & 4.37 & 759.90 & 89.98 \\
C (50) & 4.07 & 488.03 & 89.98 \\
\hline
\end{tabular}




\section{EXPERIMENTAL RESULTS}

The fall and emergency detection platform was tested with intentional falls and ADL samples (four ADLs and three types of falls), as shown in Table 1.

\section{A. Comparison of Multiple Parameter Combinations}

The fall detection sensitivity and specificity of single parameter and multiple parameter combinations of $A V_{S V M}$, $A C C_{S V M}$, and mAngle are summarized in Tables 6 and 7, respectively. In these tables, a number in parentheses means a test count of an ADL or a fall. The multiple parameter combinations such as $A C C_{S V M} \wedge A V_{S V M} \wedge$ mAngle mean that all of the parameters were above certain thresholds, as shown in Table 2. Further, $d-A C C_{S V M}$ and $d$-mAngle denote that $A C C_{S V M}$ and $m A n g l e$ are outside the time interval, as shown in Lines 2 and 3 of the fall detection algorithm, respectively. When using the single parameters $A V_{S V M}$ and $A C C_{S V M}$, the algorithm achieves high sensitivities (100\%), but low specificities (below 50\%). In the multiple parameter combinations not containing $d-A C C_{S V M}$ and $d$-mAngle, the algorithm achieves low sensitivity and specificity because the acceleration and angular velocity during the falls or ADLs do not vary synchronously. In the multiple parameter combinations that contain $d-A C C_{S V M}$ and $d$-mAngle, the algorithm achieves relatively high sensitivity and specificity, and the combination $A V_{S V M} \wedge d-A C C_{S V M} \wedge d$-mAngle achieves the highest sensitivity (100\%) and specificity (98.98\%).

Table 6. Specificity (\%) for detecting activities of daily livings (ADLs) using single parameters and multiple parameter combinations

\begin{tabular}{|c|c|c|c|c|c|}
\hline \multirow{2}{*}{ Combination } & \multicolumn{4}{|c|}{ ADL type (no.) } & \multirow{2}{*}{$\begin{array}{l}\text { Total } \\
\text { (201) }\end{array}$} \\
\hline & $A(51)$ & B (51) & $\mathrm{C}(50)$ & D (49) & \\
\hline$A C C_{S V M}$ & 0 & 0 & 88 & 79.59 & 41.9 \\
\hline$A V_{S V M}$ & 1.96 & 41.18 & 28 & 32.65 & 25.95 \\
\hline$A C C_{S V M} \wedge A V_{S V M}$ & 5.88 & 45.1 & 100 & 93.88 & 61.21 \\
\hline$A_{C C} C_{S M} \wedge m$ Angle & 100 & 100 & 100 & 97.96 & 99.49 \\
\hline$A V_{S \backslash M} \wedge m$ Angle & 100 & 100 & 100 & 67.35 & 91.84 \\
\hline ACC $_{S M M} \wedge d-m$ Angle & 100 & 100 & 100 & 81.63 & 95.41 \\
\hline$A V_{S \bigvee M} \wedge d-m$ Angle & 100 & 100 & 100 & 48.98 & 87.24 \\
\hline$A C C_{S V M} \wedge A V_{S V M} \wedge m$ Angle & 100 & 100 & 100 & 97.96 & 99.49 \\
\hline$A C C_{S V M} \wedge A V_{S V M} \wedge d-m A n g l e$ & 100 & 100 & 100 & 95.92 & 98.98 \\
\hline$A V_{S V M} \wedge d-A C C_{S V M} \wedge m$ Angle & 100 & 100 & 100 & 97.96 & 99.49 \\
\hline$A V_{S V M} \wedge d-A C C_{S V M} \wedge d-m$ Angle & 100 & 100 & 100 & 95.92 & 98.98 \\
\hline
\end{tabular}

Table 7. Sensitivity (\%) for detecting falls using single parameter and multiple parameter combinations

\begin{tabular}{lcccc}
\hline \multirow{2}{*}{ Combination } & \multicolumn{3}{c}{ Fall type (no.) } & \multirow{2}{*}{ Total } \\
\cline { 2 - 4 } & $\mathbf{A}(\mathbf{5 1})$ & $\mathbf{B}(\mathbf{5 0 )}$ & $\mathbf{C} \mathbf{( 5 1 )}$ & $\mathbf{( 1 5 2 )}$ \\
\hline$A C C_{S V M}$ & 100 & 100 & 100 & 100 \\
$A V_{S V M}$ & 100 & 100 & 100 & 100 \\
$A C C_{S V M} \wedge A V_{S V M}$ & 84.31 & 100 & 100 & 94.77 \\
$A C C_{S V M} \wedge m A n g l e$ & 37.25 & 30 & 0 & 22.42 \\
$A V_{S V M} \wedge m A n g l e$ & 37.25 & 30 & 1.96 & 23.07 \\
$A C C_{S V M} \wedge d-m A n g l e$ & 100 & 100 & 100 & 100 \\
$A V_{S V M} \wedge d-m A n g l e$ & 100 & 100 & 100 & 100 \\
$A C C_{S V M} \wedge A V_{S V M} \wedge m A n g l e$ & 29.41 & 30 & 0 & 19.8 \\
$A C C_{S V M} \wedge A V_{S V M} \wedge d-m A n g l e$ & 84.31 & 100 & 100 & 94.77 \\
$A V_{S V M} \wedge d-A C C_{S V M} \wedge m A n g l e$ & 37.25 & 30 & 0 & 22.42 \\
$A V_{S V M} \wedge d-A C C_{S V M} \wedge d-m A n g l e$ & 100 & 100 & 100 & 100 \\
\hline
\end{tabular}

\section{B. Comparison of Fall Detection Algorithms}

The fall detection algorithm was tested for four types of ADLs and three types of falls, and its performance was compared with that of two different fall detection algorithms proposed by Kangas et al. [10] and Weiss et al. [11], as shown in Tables 8 and 9. The sensitivities of the algorithms proposed by Kangas and Weiss are as high as $100 \%$ and $99.35 \%$, respectively, but the specificities are $95.31 \%$ and $32.71 \%$, respectively. The specificities of Weiss' algorithm are very low because it uses only single parameters, but those of Kangas' algorithm and the proposed algorithm are very high because they use multiple parameter combinations In ADL-D, the specificity of the proposed algorithm coupled with the accelerometer and the gyroscope is $14 \%$ higher than that of Kangas' algorithm, which uses only the

Table 8. Specificity (\%) of each algorithm for detecting activities of daily livings (ADLs)

\begin{tabular}{lccccc}
\hline \multirow{2}{*}{ Algorithm } & \multicolumn{5}{c}{ ADL type (no.) } \\
\cline { 2 - 5 } & A (51) & B (51) & C (50) & D (49) & \\
\hline Kangas et al. [10] & 100 & 100 & 100 & 81.63 & 95.41 \\
Weiss et al. [11] & 0 & 0 & 88 & 42.86 & 32.71 \\
Proposed & 100 & 100 & 100 & 95.92 & 98.98 \\
\hline
\end{tabular}

Table 9. Sensitivity (\%) of each algorithm for detecting falls

\begin{tabular}{lcccc}
\hline \multirow{2}{*}{ Algorithm } & \multicolumn{3}{c}{ Fall type (no.) } & \multirow{2}{*}{ Total (152) } \\
\cline { 2 - 4 } & A (51) & B (50) & C (51) & \\
\hline Kangas et al. [10] & 100 & 100 & 100 & 100 \\
Weiss et al. [11] & 98.04 & 100 & 100 & 99.35 \\
Proposed & 100 & 100 & 100 & 100 \\
\hline
\end{tabular}


accelerometer, because moving into a lying posture induces both angular velocity and acceleration.

However, because the test subjects are limited to persons aged 25 to 28 years old in these experiments, elderly persons should be tested in the future to obtain the threshold for old persons, and because the threshold may change according to the test subjects, a more effective classifying method such as machine learning, rather than a simple threshold, should be developed.

\section{Results of the Proposed Emergency Alarm Algorithm}

The emergency alarm system was tested with three types of falls. The parameters, $m A C C_{p p}, m A V_{p p}$, and $m A$ ngle, were used for monitoring the fallen elderly person's movements. The emergency detection sensitivity for three types of falls was $100 \%$. This algorithm achieved the best possible emergency detection sensitivity.

\section{CONCLUSION}

We proposed an emergency monitoring system equipped with a sensor node for detecting falls suffered by the elderly, and the resulting emergencies, using a newly developed fall detection algorithm. The sensor node consisted of a TI CC2530 chip and three types of sensors: an ECG, an accelerometer, and a gyroscope. The fall detection algorithm used multiple parameter combinations in which all of the parameters, calculated using accelerations in three directions and angular velocities in two directions, were above a certain threshold within a time period. In addition, we proposed an emergency detection algorithm that monitors the movements of the fallen elderly person after the fall is detected. The results showed that the proposed fall detection algorithm achieved $100 \%$ sensitivity and $98.98 \%$ specificity and that it could distinguish falls from ADLs. Further, when a fall was detected, the emergency detection sensitivity was $100 \%$, suggesting that the proposed fall and emergency detection system provided an effective automatic fall detection tool and emergency alarm.

One limitation of this study is that the fall detection algorithm was tested on young subjects falling under simulated conditions. Further research is required to test the performance of the proposed algorithm for detecting actual falls suffered by the elderly and to develop a more effective classifying algorithm such as machine learning, rather than a simple threshold method, and to evaluate the power consumption of the fall detection sensor node for testing the power consumption of the proposed fall detection algorithm.

\section{REFERENCES}

[ 1 ] I. Neild, D. J. T. Heatley, R. S. Kalawsky, and P. A. Bowman, "Sensor networks for continuous health monitoring," BT Technology Journal, vol. 22, no. 3, pp. 130-139, 2004.

[ 2 ] M. H. Park, J. C. Ha, I. H. Shin, H. G. Kim, S. Y. Lee, J. H. Cho, H. R. Kim, E. J. Kim, J. S. Kim, M. H. Park, J. M. Lee, E. J. Kim, Y. M. Yim, G. R. Hong, and J. A. Song, Senior Survey 2008: Life and Welfare Service Needs of the Elderly in Korea. Seoul: Ministry for Health and Welfare, 2009.

[ 3 ] L. Z. Rubenstein and K. R. Josephson, "Falls and their prevention in elderly people: what does the evidence show?," Medical Clinics of North America, vol. 90, no. 5, pp. 807-824, 2006.

[ 4 ] B. J. Vellas, S. J. Wayne, L. J. Romero, R. N. Baumgartner, and P. J. Garry, "Fear of falling and reduction of mobility in elderly fallers,” Age and Ageing, vol. 26, no. 3, pp. 189-193, 1997.

[5] E. J. Porter, "Wearing and using personal emergency response system buttons," Journal of Gerontological Nursing, vol. 31, no. 10, pp. 26-33, 2005.

[ 6] A. K. Bourke, J. V. O'Brien, and G. M. Lyons, "Evaluation of a threshold-based tri-axial accelerometer fall detection algorithm," Gait \& Posture, vol. 26, no. 2, pp. 194-199, 2007.

[ 7 ] M. Kangas, A. Konttila, I. Winblad, and T. Jamsa, "Determination of simple thresholds for accelerometry-based parameters for fall detection," in Proceeding of the 29th Annual International Conference of the IEEE Engineering in Medicine and Biology Society, Lyon, France, pp. 1367-1370, 2007.

[ 8 ] M. Kangas, A. Konttila, P. Lindgren, I. Winblad, and T. Jamsa, "Comparison of low-complexity fall detection algorithms for body attached accelerometers,” Gait \& Posture, vol. 28, no. 2, pp. 285291, 2008.

[ 9 ] P. K. Chao, H. L. Chan, F. T. Tang, Y. C. Chen, and M. K. Wong, "A comparison of automatic fall detection by the cross-product and magnitude of tri-axial acceleration,” Physiological Measurement, vol. 30, no. 10, pp. 1027-1037, 2009.

[10] M. Kangas, I. Vikman, J. Wiklander, P. Lindgren, L. Nyberg, and T. Jamsa, "Sensitivity and specificity of fall detection in people aged 40 years and over," Gait \& Posture, vol. 29, no. 4, pp. 571574, 2009.

[11] A. Weiss, I. Shimkin, N. Giladi, and J. M. Hausdorff, “Automated detection of near falls: algorithm development and preliminary results,” BMC Research Notes, vol. 3, p. 62, 2010.

[12] A. K. Bourke and G. M. Lyons, “A threshold-based fall-detection algorithm using a bi-axial gyroscope sensor,” Medical Engineering \& Physics, vol. 30, no. 1, pp. 84-90, 2008.

[13] Q. Li, J. A. Stankovic, M. A. Hanson, A. T. Barth, J. Lach, and G. Zhou, "Accurate, fast fall detection using gyroscopes and acelerometer-derived posture information,” in Proceeding of the 6th International Workshop Wearable and Implantable Body Sensor Networks, Berkeley: CA, pp. 138-143, 2009.

[14] M. L. Lehrman, M. D. Halleck, and E. L. Massman, "System and method for detecting motion of a body motion," Patent US 7095331, 2006. 
[15] M. A. Clifford, R. L. Borras, and L. Gomez, "System and method for human body fall detect detection,” Patent US 7248172, 2007.

[16] LabVIEW [Internet], Available: http://www.ni.com/labview/.

[17] M. Raju, "Heart-rate and EKG monitor using the msp430fg439," Texas Instruments, Dallas, TX, Application Report SLAA280A, 2007.
[18] K. Tuck, "Implementing auto-zero calibration technique for accelerometers," Freescale Semiconductor, Austin, TX, AN3447, 2007.

[19] K. Tuck, “Tilt sensing using linear accelerometers,” Freescale Semiconductor, Austin, TX, AN3461, 2007.

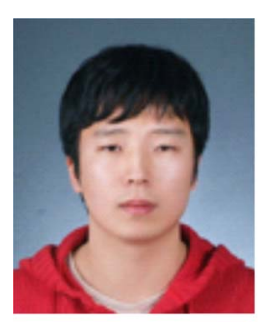

\section{Yun Jae Yi}

received a B.S. in Information and Control Engineering and M.S. in Signal Processing of the Bio EnvironmentInformation Communication Graduate School at Hankyong National University, Anseong, Korea, in 2009 and 2011, respectively. He is now a research engineer with LaonPeople Inc., Seongnam, Korea.

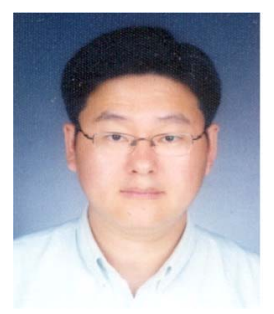

\section{Yun Seop Yu}

received B.S., M.S., and Ph. D. degrees from the Department of Electronics Engineering of Korea University, Seoul, Korea, in 1995, 1997, and 2001, respectively. From 2001 to 2002, he worked as a Guest Researcher at the Electronics and Electrical Engineering Laboratory of NIST, Gaithersburg, MD, USA. He iis now an Associate Professor with the Department of Electrical, Electronic and Control Engineering, Hankyong National University, Anseong, Korea. His main research interests are in the fields of the modeling of various nano devices for efficient circuit simulation, and future memory, logic, and sensor designs using those devices. He has authored and coauthored 60 refereed international journal papers. He is also interested in the fabrication and characterization of various nano devices, as well as applications thereof such as future memory, logic, and sensors. 